\title{
A Condition Number Analysis of a Line-Surface Intersection Algorithm*
}

\author{
Gun Srijuntongsiri ${ }^{\dagger}$ Stephen A. Vavasis ${ }^{\ddagger}$
}

September 11, 2018

\begin{abstract}
We propose an algorithm based on Newton's method and subdivision for finding all zeros of a polynomial system in a bounded region of the plane. This algorithm can be used to find the intersections between a line and a surface, which has applications in graphics and computer-aided geometric design. Our analysis shows that the running time of the algorithm is bounded in terms of the condition number of the polynomial's zeros. We argue that, in contrast, some other well known algorithms for this problem similarly based on Newton and subdivision may in certain cases require excessive computation even for well conditioned problems.
\end{abstract}

\section{Introduction}

The problem of line-surface intersection has many applications in areas such as geometric modeling, robotics, collision avoidance, manufacturing simulation, scietific visualization, and computer graphics. It is also a basis for considering intersections between more complicated objects. This article deals with intersections of a line and a parametric surface. The parametric method of surface representation is a very convenient way of approximating and designing curved surfaces, and computation using parametric representation is often much more efficient than other types of surface representations.

\footnotetext{
*Supported in part by NSF DMS 0434338 and NSF CCF 0085969.

$\dagger 4130$ Upson Hall, Cornell University, Ithaca, NY 14853. Email: gunsri@cs.cornell.edu.

$\ddagger 4130$ Upson Hall, Cornell University, Ithaca, NY 14853. Email: vavasis@cs.cornell.edu.
} 
Typically, intersection problems reduce to solving systems of nonlinear equations. Subdivision methods introduced by Whitted [21, 15] were the first to be used for this problem. In these methods, a simple shape such as rectangular box or sphere is used to bound the surface and is tested whether the line intersects the bounding volume. If it does, the surface patch is subdivided, and the bounding volumes are found for each subpatch. The process repeats until no bounding volumes intersect the line or the volumes are smaller than a specified size where the intersection between such volumes and the line are taken as the intersections between the surface and the line. Subdivision methods are robust and simple, but normally not efficient when high accuracy of the solutions are required. They also cannot indicate if there are more than one zero inside the remaining subdomains.

Regardless, a variation of subdivision methods known as Bézier clipping by Nishita et al. should be noted for its efficient subdivision [13]. For a non-rational Bézier surface, Bézier clipping uses the intersection between the convex hull of the orthographic projection of the surface along the line and a parameter axis to determine the regions which do not contain any intersections before subdividing the remaining region. Sherbrooke and $\mathrm{Pa}-$ trikalakis generalizes Bézier clipping to a zero-finding algorithm for an $n$ dimensional nonlinear polynomial system called Projected Polyhedron (PP) algorithm [16].

On the numerical side, Kajiya [10] proposes a method for intersecting a line with a bicubic surface based on algebraic geometry without subdivisions. His method is robust and simple. However, it is too costly to extend to higher degree polynomials. Jónsson and Vavasis [9] introduce an algorithm for solving systems of two polynomials in two variables using Macaulay resultant matrices. They also analyze the accuracy of the computed zeros in term of the conditioning of the problem.

Another approach is to combine a subdivision method with a Newtontype method, using the latter to find the solutions of the resulted system of equations after subpatches pass some criteria. The advantages of Newton's method are its quadratic convergence and simplicity in implementation, but it requires a good initial approximation to converge and does not guarantee that all zeros have been found. To remedy these shortcomings, Toth [19] uses the result from interval analysis to determine the "safe regions", where a Newton-like method starting from any point in them are guaranteed to converge. He tests each patch if it is a safe region and if its axis-aligned bounding box intersects the line. If neither is true, the patch is subdivided 
recursively. Lischinski and Gonczarowski [12] propose an improvement to Toth's algorithm specific to scene-rendering in computer graphics by utilizing ray and surface coherences.

In contrast, other researchers develop methods to estimate good initial points for Newton's method rather than test for convergence of each choice of initial points. These methods use tighter bounding volumes and subdivide the surface adaptively until subpatches are flat enough, that is, until they are close enough to the bounding volumes. Then the intersection between the bounding volume and the line is chosen as the initial point for Newton's method. Examples of methods in this category are [1, 6, 14, 17, 22. There is also the ray-tracing algorithm by Wang et al. that combines Newton's method and Bézier clipping together 20.

Among the known methods in the literature, Toth's is the most similar one to our proposed algorithm. Ours is based on Newton's method, and it tests for the convergence of initial points before performing Newton's iteration. The critical differences between our algorithm and Toth's are the choice of convergence test and how the domains for such test are selected. Toth's uses the Krawczyk-Moore test and another unnamed test based on interval analysis. Unlike Toth, we choose the Kantorovich test because it also tells us whether Newton's method converges quadratically for the initial point in question in addition to whether it converges at all. The tests in Toth's, on the other hand, guarantee only linear convergence for the simple Newton iteration - a variation of Newton's method where the Jacobian of the initial point is used in place of the Jacobian of the current point in every iteration. With the Kantorovich test, we can choose to hold off Newton's method until quadratic convergence is assured.

The difference in the choice of domains for the convergence test is due to the fact that algorithms using the subpatches as the domains (e.g., Toth's) may exhibit undesirable behavior when an intersection lies on the border of a subpatch, which is not necessarily on or near the border of the original surface. The convergence tests in Toth's algorithm cannot detect the safe regions for zeros on the border of the domain. For example, consider the function $f(u, v)=\left(u^{2}-.25, v-.8\right)^{T}$ whose zeros are $(.5, .8)$ and $(-.5, .8)$. The patch $\{(u, v): .5 \leq u \leq .5+\epsilon, a \leq v \leq b\}$ does not pass either of the tests for any $\epsilon>0$ and any $a \leq .8 \leq b$ although the patch $\{(u, v): .45 \leq u \leq$ $.8,0 \leq v \leq 1\}$ does pass the Krawczyk-Moore test. This results in excessive subdivisions by Toth's algorithm. Our algorithm, on the other hand, chooses the domain in the manner that avoids this difficulty. In particular, our main 
theorem (Theorem 6.6) shows that the amount of subdivisions performed by our algorithm depends only on the condition number of the problem instance. Toth's algorithm does not have this property as its efficiency also depends on the boundaries of the subpatches, which are independent of the conditioning of the instance. To the best of our knowledge, there is no previous algorithm in this class whose running time has been bounded in terms of the condition of the underlying problem instance, and we are not sure whether such an analysis is possible for previous algorithms.

The notion of bounding the running time of an iterative method in terms of the condition number of the instance is an old one, with the most notable example being the condition-number bound of conjugate gradient (see Chapter 10 of [8]). This approach has also been used in interior-point methods for linear programming [7] and Krylov-space eigenvalue computation [18].

\section{The theorem of Kantorovich}

Denote the closed ball centered at $x$ with radius $r>0$ with

$$
\bar{B}(x, r)=\left\{y \in \mathbb{R}^{n}:\|y-x\| \leq r\right\},
$$

and denote $B(x, r)$ as the interior of $\bar{B}(x, r)$. Kantorovich's theorem in affine invariant form is

Theorem 2.1 (Kantorovich, affine invariant form [2, 11]). Let $f: D \subseteq$ $\mathbb{R}^{n} \rightarrow \mathbb{R}^{n}$ be differentiable in the open convex set $D$. Assume that for some point $x^{0} \in D$ the Jacobian $f^{\prime}\left(x^{0}\right)$ is invertible with

$$
\left\|f^{\prime}\left(x^{0}\right)^{-1} f\left(x^{0}\right)\right\| \leq \eta
$$

Let there be a Lipschitz constant $\omega>0$ for $f^{\prime}\left(x^{0}\right)^{-1} f^{\prime}$ such that

$$
\left\|f^{\prime}\left(x^{0}\right)^{-1}\left(f^{\prime}(x)-f^{\prime}(y)\right)\right\| \leq \omega \cdot\|x-y\| \text { for all } x, y \in D .
$$

If $h=\eta \omega \leq 1 / 2$ and $\bar{B}\left(x^{0}, \rho_{-}\right) \subseteq D$, where

$$
\rho_{-}=\frac{1-\sqrt{1-2 h}}{\omega}
$$

then $f$ has a zero $x^{*}$ in $\bar{B}\left(x^{0}, \rho_{-}\right)$. Moreover, this zero is the unique zero of $f$ in $\left(\bar{B}\left(x^{0}, \rho_{-}\right) \cup B\left(x^{0}, \rho_{+}\right)\right) \cap D$ where

$$
\rho_{+}=\frac{1+\sqrt{1-2 h}}{\omega}
$$


and the Newton iterates $x^{k}$ with

$$
x^{k+1}=x^{k}-f^{\prime}\left(x^{k}\right)^{-1} f\left(x^{k}\right)
$$

are well-defined, remain in $\bar{B}\left(x^{0}, \rho_{-}\right)$, and converge to $x^{*}$. In addition,

$$
\left\|x^{*}-x^{k}\right\| \leq \frac{\eta}{h}\left(\frac{(1-\sqrt{1-2 h})^{2^{k}}}{2^{k}}\right), k=0,1,2, \ldots
$$

We call $x^{0}$ a fast starting point if the sequence of Newton iterates starting from it converges to a solution $x^{*}$ and (1D) is satisfied with $h \leq 1 / 4$, which implies quadratic convergence of the iterates starting from $x^{0}$. The Kantorovich's theorem also holds for complex functions [5].

\section{The Kantorovich-Test Subdivision algorithm}

For this section, let $B_{i, m}(u)$ denote the Bernstein basis polynomial $B_{i, m}(u)=$ $\left(\begin{array}{c}m \\ i\end{array}\right)(1-u)^{m-i} u^{i}$. Let $S$ be a two-dimensional Bézier surface embedded in $\mathbb{R}^{3}$ parametrized by

$$
\bar{f}(u, v)=\sum_{i=0}^{m} \sum_{j=0}^{n} \overline{\mathbf{b}}_{i j} B_{i, m}(u) B_{j, n}(v), \quad 0 \leq u, v \leq 1,
$$

where $\overline{\mathbf{b}}_{i j} \in \mathbb{R}^{3}(i=0,1, \ldots, m ; j=0,1, \ldots, n)$ denote the control points. Define a line

$$
L=\{\mathbf{p}+t \mathbf{d}: t \in \mathbb{R}\},
$$

where $\mathbf{p}, \mathbf{d} \in \mathbb{R}^{3}, \mathbf{d} \neq \mathbf{0}$. The intersection points between $S$ and $L$ are the solutions of the polynomial system

$$
\bar{f}(u, v)-(\mathbf{p}+t \mathbf{d})=0 .
$$

The system (22) can be reduced to a system of two equations and two unknowns. To show this, we first break (2) into its component parts

$$
\begin{aligned}
& \bar{f}_{1}(u, v)-p_{1}-t d_{1}=0, \\
& \bar{f}_{2}(u, v)-p_{2}-t d_{2}=0, \\
& \bar{f}_{3}(u, v)-p_{3}-t d_{3}=0 .
\end{aligned}
$$


Here, the subscript $i$ denotes the $i$ th coordinate of the point in three-dimensional space. Assuming $\left|d_{1}\right| \geq \max \left\{\left|d_{2}\right|,\left|d_{3}\right|\right\}$, we have the equivalent system

$$
\begin{aligned}
& d_{1}\left(\bar{f}_{2}(u, v)-p_{2}\right)-d_{2}\left(\bar{f}_{1}(u, v)-p_{1}\right)=0 \\
& d_{1}\left(\bar{f}_{3}(u, v)-p_{3}\right)-d_{3}\left(\bar{f}_{1}(u, v)-p_{1}\right)=0 .
\end{aligned}
$$

By noting that $\sum_{i=0}^{m} \sum_{j=0}^{n} B_{i, m}(u) B_{j, n}(v)=1$, it is seen that (3) is equivalent to

$$
f(u, v) \equiv \sum_{i=0}^{m} \sum_{j=0}^{n} \mathbf{b}_{i j} B_{i, m}(u) B_{j, n}(v)=0,
$$

where

$$
\mathbf{b}_{i j}=\left(\begin{array}{c}
d_{1}\left(\left[\bar{b}_{i j}\right]_{2}-p_{2}\right)-d_{2}\left(\left[\bar{b}_{i j}\right]_{1}-p_{1}\right) \\
d_{1}\left(\left[\bar{b}_{i j}\right]_{3}-p_{3}\right)-d_{3}\left(\left[\bar{b}_{i j}\right]_{1}-p_{1}\right)
\end{array}\right)
$$

Here, $\left[\bar{b}_{i j}\right]_{k}$ denotes the $k$ th coordinate of $\bar{b}_{i j}$. We choose to solve (44) rather than (2) because $f(u, v)$ is a polynomial in Bernstein basis, a fact that is to be exploited by our algorithm.

Before we detail our algorithm, we define notation and crucial quantities that are used by the algorithm and its analysis. Since the parametric domain of the surface in consideration is square, our algorithm uses the infinity norm for all of its norm computation. Therefore, for the rest of this paper, the notation $\|\cdot\|$ is used to refer specifically to infinity norm. The closed ball $X=\bar{B}(x, r)$ with respect to infinity norm is square, whose width is defined to be $2 r$. We use the notation $f(x)=f(u, v)$ where $x=(u, v)$ is a point in two-dimensional parametric space.

For a given zero $x^{*}$ of polynomial $f$, let $\omega_{*}\left(x^{*}\right)$ and $\rho_{*}\left(x^{*}\right)$ be quantities satisfying the conditions that, first, $\omega_{*}\left(x^{*}\right)$ is the smallest Lipschitz constant for $f^{\prime}\left(x^{*}\right)^{-1} f^{\prime}$, i.e.,

$$
\left\|f^{\prime}\left(x^{*}\right)^{-1}\left(f^{\prime}(x)-f^{\prime}(y)\right)\right\| \leq \omega_{*}\left(x^{*}\right) \cdot\|x-y\| \text { for all } x, y \in \bar{B}\left(x^{*}, \rho_{*}\left(x^{*}\right)\right)
$$

and, second,

$$
\rho_{*}\left(x^{*}\right)=\frac{2}{\omega_{*}\left(x^{*}\right)} .
$$

Since $\omega_{*}\left(x^{*}\right)$ is nondecreasing as $\rho_{*}\left(x^{*}\right)$ increases in (5) but $\rho_{*}\left(x^{*}\right)$ is decreasing as $\omega_{*}\left(x^{*}\right)$ increases in (6) $)$, there exists a unique pair $\left(\omega_{*}\left(x^{*}\right), \rho_{*}\left(x^{*}\right)\right)$ satisfying the above conditions, and this pair can be obtained by binary search. When more than one function is being discussed, we use $\omega_{*}^{f}\left(x^{*}\right)$ to denote 
$\omega_{*}\left(x^{*}\right)$ of the function $f$. Approximation to these two quantities, $\omega_{*}\left(x^{*}\right)$ and $\rho_{*}\left(x^{*}\right)$, are computed and made use of by the algorithm.

For clarity, we simply abbreviate $\omega_{*}\left(x^{*}\right)$ and $\rho_{*}\left(x^{*}\right)$ as $\omega_{*}$ and $\rho_{*}$, respectively, throughout the rest of this article when it is clear from the context to which $x^{*}$ the quantities belong.

Straightforward application of the affine invariant form of Kantorovich's theorem with $x^{0}=x^{*}$ and $D=\bar{B}\left(x^{*}, \rho_{*}\left(x^{*}\right)\right)$ yields the result that $x^{*}$ is the unique zero of $f$ in $\bar{B}\left(x^{*}, \rho_{*}\left(x^{*}\right)\right)$. In fact, the above definitions of $\omega_{*}\left(x^{*}\right)$ and $\rho_{*}\left(x^{*}\right)$ are chosen such that the ball that is guaranteed by the Kantorovich's theorem to contain no other zeros than $x^{*}$ is the largest possible.

Another quantity of interest is $\omega_{D^{\prime}}$, which is defined as the smallest nonnegative constant $\omega$ satisfying

$$
\begin{array}{ll}
\left\|f^{\prime}\left(x^{*}\right)^{-1}\left(f^{\prime}(y)-f^{\prime}(z)\right)\right\| \leq \omega \cdot\|y-z\|, & y, z \in D^{\prime}, x^{*} \in[0,1]^{2} \\
& \text { satisfying } f\left(x^{*}\right)=0
\end{array}
$$

where

$$
D^{\prime}=[-\epsilon(m, n), 1+\epsilon(m, n)]^{2}
$$

and

$$
\epsilon(m, n)=\frac{m^{m} n^{n}}{m^{m} n^{n}+4 \sqrt{5}-9} .
$$

Denote $\omega_{f}$ as the maximum of $\omega_{D^{\prime}}$ and all $\omega_{*}\left(x^{*}\right)$

$$
\omega_{f}=\max \left\{\omega_{D^{\prime}}, \max _{x^{*} \in \mathbb{C}^{2}: f\left(x^{*}\right)=0} \omega_{*}\left(x^{*}\right)\right\}
$$

Finally, define the condition number of $f$ to be

$$
\operatorname{cond}(f)=\max \left\{\omega_{f}, \max _{x^{*} \in \mathbb{C}^{2}: f\left(x^{*}\right)=0, y \in[0,1]^{2}}\left\|f^{\prime}\left(x^{*}\right)^{-1} f^{\prime}(y)\right\|\right\} .
$$

Note that in (7), $x^{*}$ is restricted to zeros in $[0,1]^{2}$ whereas in (9), $x^{*}$ ranges over all complex zeros of $f$. We defer the discussion of why (9) is a reasonable condition number until after the description of our algorithm.

We define the Kantorovich test on a region $X=\bar{B}\left(x^{0}, r\right)$ as the application of the Kantorovich's Theorem on the point $x^{0}$ using $\bar{B}\left(x^{0}, 2 \epsilon(m, n) r\right)$ as the domain $D$ in the statement of the theorem and $\left\|f^{\prime}\left(x^{0}\right)^{-1} f\left(x^{0}\right)\right\|$ as $\eta$. For $\omega$, we instead use $\hat{\omega} \geq \omega$, where $\hat{\omega}$ is defined by (11) below. The region $X$ passes the Kantorovich test if $\eta \hat{\omega} \leq 1 / 4$ and $\bar{B}\left(x^{0}, \rho_{-}\right) \subseteq D^{\prime}$, which implies that $x^{0}$ is a fast starting point. 
The other test our algorithm uses is the exclusion test. For a given region $X$, let $\hat{f}_{X}$ be the polynomial in Bernstein basis that reparametrizes with $[0,1]^{2}$ the Bézier surface defined by $f$ over $X$. Let $\left\{\hat{\mathbf{b}}_{i j}\right\}$ be the control points of $\hat{f}_{X}$. The region $X$ passes the exclusion test if the convex hull of $\left\{\hat{\mathbf{b}}_{i j}\right\}$ excludes the origin. This implies that there are no zeros of $f$ in $X$ by the convex hull property of Bézier surfaces.

We now proceed to describe our algorithm, the Kantorovich-Test Subdivision algorithm or KTS in short.

\section{Algorithm KTS:}

- Let $Q$ be a queue with $[0,1]^{2}$ as its only entry. Set $S=\emptyset$.

- Repeat until $Q=\emptyset$

1. Let $X$ be the patch at the front of $Q$. Remove $X$ from $Q$.

2. If $X \nsubseteq X_{S}$ for all $X_{S} \in S$,

- Perform the exclusion test on $X=\bar{B}\left(x^{0}, r\right)$

- If $X$ fails the exclusion test,

(a) Perform the Kantorovich test on $X$

(b) If $X$ passes the Kantorovich test,

i. Perform Newton's method starting from $x^{0}$ to find a zero $x^{*}$.

ii. If $x^{*} \notin X_{S}$ for any $X_{S} \in S$ (i.e., $x^{*}$ has not been found previously),

* Compute $\rho_{*}\left(x^{*}\right)$ and its associated $\omega_{*}\left(x^{*}\right)$ by binary search.

* Set $S=S \cup\left\{\bar{B}\left(x^{*}, \rho_{*}\left(x^{*}\right)\right)\right\}$.

(c) Subdivide $X$ along both $u$ and $v$-axes into four equal subregions. Add these subregions to the end of $Q$.

A few remarks are needed regarding the description of the KTS algorithm.

- The subdivision in step 2.c is performed regardless of the result of the Kantorovich test. In general, passing the Kantorovich test does not imply that there is only one zero in $X$. 
- The check that the zero found by Newton's method is not a duplicate (step 2.b.ii) is necessary since the Kantorovich test may detect a zero outside $X$.

- If the Kantorovich test is not applicable for a certain patch due to the Jacobian of the midpoint being singular, the patch is treated as if it fails the Kantorovich test.

One property of KTS is that it is affine invariant. In other words, leftmultiplying $f$ with a 2-by-2 matrix $A$ prior to executing KTS does not change its behavior. This is the main reason we define the condition number to be affine invariant. Define $g \equiv A f$. To see that our condition number is affine invariant, note that $g^{\prime}(x)^{-1} g^{\prime}(y)=\left[A f^{\prime}(x)\right]^{-1} A f^{\prime}(y)=f^{\prime}(x)^{-1} A^{-1} A f^{\prime}(y)=$ $f^{\prime}(x)^{-1} f^{\prime}(y)$ for any $x, y \in \mathbb{R}^{n}$. Therefore, $\operatorname{cond}(g)=\operatorname{cond}(f)$. In contrast, simpler condition numbers such as Lipschitz constants for $f^{\prime}$ are not affine invariant and hence are not chosen for our analysis.

\section{Implementation details}

There are two steps in the description of KTS whose implementations need further elaboration, namely the computation of Lipschitz constant in the Kantorovich test and the reparametrization required for the exclusion test. The Lipschitz constant for $f^{\prime}\left(x^{0}\right)^{-1} f^{\prime} \equiv g$ is obtained from an upper bound of the derivative of $g$

$$
g^{\prime}(x)=\left(\frac{\partial^{2} g_{i}(x)}{\partial x_{j} \partial x_{k}}\right)
$$

for all $x \in X$. Let $\hat{g} \equiv \hat{g}_{X}$ be the polynomial in Bernstein basis that reparametrizes with $[0,1]^{2}$ the Bézier surface defined by $g$ over $X$. We have that

$$
\begin{aligned}
\max _{x \in X}\left\|g^{\prime}(x)\right\| & =\max _{x \in[0,1]^{2}}\left\|\hat{g}^{\prime}(x)\right\| \\
& =\max _{x \in[0,1]^{2}} \max _{\|y\|=1}\left\|\hat{g}^{\prime}(x) y\right\| \\
& \leq \max _{x \in[0,1]^{2}} \max _{i} \sum_{j=1}^{2} \sum_{k=1}^{2}\left|\hat{g}_{i j k}^{\prime}(x)\right| \\
& \leq 4 \max _{i, j, k} \max _{x \in[0,1]^{2}}\left|\hat{g}_{i j k}^{\prime}(x)\right| .
\end{aligned}
$$


Note that each entry of $\hat{g}^{\prime}$ can be written as a polynomial in Bernstein basis. Therefore, the maximum absolute value of the coefficients of $\hat{g}_{i j k}^{\prime}$ is an upper bound of $\max _{x \in[0,1]^{2}}\left|\hat{g}_{i j k}^{\prime}(x)\right|$. Let $\hat{\omega}$ denote the Lipschitz constant computed from right-hand side of (10). That is,

$$
\hat{\omega} \equiv 4 \max _{i, j, k} \max _{x \in[0,1]^{2}}\left|\hat{g}_{i j k}^{\prime}(x)\right| .
$$

The other step requiring explanation is the reparametrization. There is more than one algorithm to compute the reparametrization with $[0,1]^{2}$ of a Bézier surface. We describe one method here. Our method makes use of a program that, given $\alpha_{i j}$ 's, $c, d, e, f, g, h, k$, and $l$, computes $\beta_{i j}$ 's satisfying

$$
\sum_{i=0}^{m} \sum_{j=0}^{n} \alpha_{i j}(c y+d)^{i}(e y+f)^{m-i}(g z+h)^{j}(k z+l)^{n-j}=\sum_{i=0}^{m} \sum_{j=0}^{n} \beta_{i j} y^{i} z^{j} .
$$

Such conversion can be done in $O\left((m n)^{2}\right)$ by generalizing Horner's rule. We leave the details of the conversion to the reader. Let $X$ denote $\{(u, v)$ : $\left.u^{0}-r \leq u \leq u^{0}+r, v^{0}-r \leq v \leq v^{0}+r\right\}$. To compute the control points $\left\{\hat{\mathbf{b}}_{i j}\right\}$ of $\left\{\hat{f}_{X}(\hat{u}, \hat{v}): 0 \leq \hat{u}, \hat{v} \leq 1\right\}$, the $[0,1]^{2}$-reparametrized surface of $\left\{f(u, v): u^{0}-r \leq u \leq u^{0}+r, v^{0}-r \leq v \leq v^{0}+r\right\}$, first substitute $u=2 r \hat{u}+u^{0}-r$ and $v=2 r \hat{v}+v^{0}-r$ into $f$, yielding

$$
\begin{aligned}
f(u, v) & =\sum_{i=0}^{m} \sum_{j=0}^{n}\left(\begin{array}{c}
m \\
i
\end{array}\right)\left(\begin{array}{c}
n \\
j
\end{array}\right) \mathbf{b}_{i j} u^{i}(1-u)^{m-i} v^{j}(1-v)^{n-j} \\
& =\sum_{i=0}^{m} \sum_{j=0}^{n}\left(\begin{array}{c}
m \\
i
\end{array}\right)\left(\begin{array}{c}
n \\
j
\end{array}\right) \mathbf{b}_{i j}\left(2 r \hat{u}+u^{0}-r\right)^{i}\left(1-\left(2 r \hat{u}+u^{0}-r\right)\right)^{m-i} . \\
& =\sum_{i=0}^{m} \sum_{j=0}^{n} \beta_{i j} \hat{u}^{i} \hat{v}^{j},
\end{aligned}
$$

where (13) is the standard form polynomial of $\hat{f}_{X}$ and is obtained from (12) by the conversion program mentioned above. Having $\beta_{i j}$ 's, use the conversion program to find $\gamma_{i j}$ 's such that

$$
\sum_{i=0}^{m} \sum_{j=0}^{n} \beta_{i j} \tilde{u}^{i}(\tilde{u}+1)^{m-i} \tilde{v}^{j}(\tilde{v}+1)^{n-j}=\sum_{i=0}^{m} \sum_{j=0}^{n} \gamma_{i j} \tilde{u}^{i} \tilde{v}^{j}
$$


Dividing both sides of (14) by $(\tilde{u}+1)^{m}(\tilde{v}+1)^{n}$ yields

$$
\sum_{i=0}^{m} \sum_{j=0}^{n} \beta_{i j}\left(\frac{\tilde{u}}{\tilde{u}+1}\right)^{i}\left(\frac{\tilde{v}}{\tilde{v}+1}\right)^{j}=\sum_{i=0}^{m} \sum_{j=0}^{n} \gamma_{i j} \frac{\tilde{u}^{i}}{(\tilde{u}+1)^{m}} \frac{\tilde{v}^{j}}{(\tilde{v}+1)^{n}}
$$

By substituting $\tilde{u}=\hat{u} /(1-\hat{u})$ and $\tilde{v}=\hat{v} /(1-\hat{v})$ into (15), it is revealed that

$$
\sum_{i=0}^{m} \sum_{j=0}^{n} \beta_{i j} \hat{u}^{i} \hat{v}^{j}=\sum_{i=0}^{m} \sum_{j=0}^{n} \gamma_{i j} \hat{u}^{i}(1-\hat{u})^{m-i} \hat{v}^{j}(1-\hat{v})^{n-j} .
$$

Therefore, $\hat{\mathbf{b}}_{i j}=\gamma_{i j} /(C(m, i) C(n, j))$ are the control points of $\hat{f}_{X}$ where $C(m, i)=\left(\begin{array}{c}m \\ i\end{array}\right)$.

\section{Significance of our Condition Number}

We now discuss the significance of (9) to the conditioning of the problem. In particular, we attempt to justify that the efficiency of any algorithm in the same class as KTS is dependent on (9). This class of algorithms being considered includes any algorithm that (i) isolates unique zeros with subdivision before finding them and (ii) uses a convex hull test of a subpatch, i.e., excludes any patch $X$ satisfying $0 \notin \operatorname{conv}(f(X))$ from further consideration. This class also includes algorithms that compute the convex hull from points on a subpatch as opposed to its control points.

We first show that, for any given zero $x^{*}$ of an arbitrary $f$, there is a function $\bar{f}$ such that $x^{*}$ is also a zero of $\bar{f}, f^{\prime}\left(x^{*}\right)=\bar{f}^{\prime}\left(x^{*}\right), \omega_{*}^{f}\left(x^{*}\right)=\omega_{*}^{\bar{f}}\left(x^{*}\right)$, and $\bar{f}$ has another zero $y^{*}$ with $\left\|y^{*}-x^{*}\right\|=\rho_{*}\left(x^{*}\right)$. Since the Kantorovich test uses only the function value, its first derivative, and the Lipschitz constant, all of which are the same for $f$ and $\bar{f}$ at $x^{*}$, the functions $f$ and $\bar{f}$ are identical from the perspective of the Kantorovich test applied to $x^{*}$. Therefore, $\rho_{*}\left(x^{*}\right)$ is a reasonable number that quantifies the distance between $x^{*}$ and its nearest other zero barring the usage of additional information. Consequently, $\omega_{f}$, which is greater than or equal to $\omega_{*}\left(x^{*}\right)=2 / \rho_{*}\left(x^{*}\right)$ for all zeros $x^{*}$ of $f$, describes the distance between the closest pairs of zeros of $f$. Therefore, the efficiency of any algorithm that isolates unique zeros is dependent on $\omega_{f}$.

We now show how to construct the function $\bar{f}$ with the above properties. Let $x^{*}=\left(u^{*}, v^{*}\right), f^{\prime}\left(x^{*}\right)=\left[\alpha_{1}, \alpha_{2} ; \alpha_{3}, \alpha_{4}\right]$, and $\omega_{*}^{f}\left(x^{*}\right)=\omega$. The definition of 
$\bar{f}$ depends on the relationship between the absolute values of $\alpha_{3}$ and $\alpha_{4}$. If $\left|\alpha_{4}\right| \geq\left|\alpha_{3}\right|$

$$
\bar{f}(u, v)=\left(\begin{array}{c}
\frac{\omega\left(\alpha_{1} \alpha_{4}-\alpha_{2} \alpha_{3}\right)}{2 \alpha_{4}}\left(u-u^{*}\right)^{2}+\alpha_{1}\left(u-u^{*}\right)+\alpha_{2}\left(v-v^{*}\right) \\
\alpha_{3}\left(u-u^{*}\right)+\alpha_{4}\left(v-v^{*}\right)
\end{array}\right) .
$$

Otherwise,

$$
\bar{f}(u, v)=\left(\begin{array}{c}
\alpha_{1}\left(u-u^{*}\right)+\frac{\omega\left(\alpha_{1} \alpha_{4}-\alpha_{2} \alpha_{3}\right)}{2 \alpha_{3}}\left(v-v^{*}\right)^{2}+\alpha_{2}\left(v-v^{*}\right) \\
\alpha_{3}\left(u-u^{*}\right)+\alpha_{4}\left(v-v^{*}\right)
\end{array}\right) .
$$

It is straightforward to verify that $\bar{f}\left(x^{*}\right)=0, \bar{f}^{\prime}\left(x^{*}\right)=f^{\prime}\left(x^{*}\right)$, and $\omega_{*}^{\bar{f}}\left(x^{*}\right)=\omega$. We now show that $\left\|y^{*}-x^{*}\right\|=\rho_{*}\left(x^{*}\right)$ for the case where $\left|\alpha_{4}\right| \geq\left|\alpha_{3}\right|$. The other case can be verified in the same manner. Let $y^{*}=\left(u^{*}+\Delta u, v^{*}+\Delta v\right)$. Substituting $y^{*}$ into (17) and setting it to zero yields

$$
g\left(u^{*}+\Delta u, v^{*}+\Delta v\right)=\left(\begin{array}{c}
\frac{\omega\left(\alpha_{1} \alpha_{4}-\alpha_{2} \alpha_{3}\right)}{2 \alpha_{4}}(\Delta u)^{2}+\alpha_{1} \Delta u+\alpha_{2} \Delta v \\
\alpha_{3} \Delta u+\alpha_{4} \Delta v
\end{array}\right)=\left(\begin{array}{l}
0 \\
0
\end{array}\right) .
$$

Solving (18) yields

$$
\begin{aligned}
\Delta u & =-\frac{2}{\omega}, \\
\Delta v & =\frac{\alpha_{3}}{\alpha_{4}} \cdot \frac{2}{\omega} .
\end{aligned}
$$

Since $\left|\alpha_{4}\right| \geq\left|\alpha_{3}\right|$ and $\rho_{*}\left(x^{*}\right)=2 / \omega,\left\|y^{*}-x^{*}\right\|=\rho_{*}\left(x^{*}\right)$.

The other term in our condition number, $\max _{x^{*} \in \mathbb{C}^{2}: f\left(x^{*}\right)=0, y \in[0,1]^{2}}\left\|f^{\prime}\left(x^{*}\right)^{-1} f^{\prime}(y)\right\|$, relates to the convex hull test - the test to determine whether the bounding convex hull of a subpatch contains the origin. We show that there exists a function $f$ such that a patch $B\left(x^{0}, r\right)$ where $x^{0}$ is relatively close to a zero fails the convex hull test if $r \geq O(1 / \operatorname{cond}(f))$. Denote $x^{0}=\left(u^{0}, v^{0}\right)$. Define the complex function $g(z)=\left(z-\left(u^{0}-\epsilon-i \epsilon\right)\right) \cdot\left(z-\left(u^{0}+\epsilon-i \epsilon\right)\right)$, where $\epsilon \in \mathbb{R}$ and $0<\epsilon<1$. Consider the following function

$$
f(u, v)=\left(\begin{array}{c}
\operatorname{Re}(g(u+i v)) \\
\operatorname{Im}(g(u+i v))
\end{array}\right)
$$

where $\operatorname{Re}(z)$ and $\operatorname{Im}(z)$ denote the real and imaginary parts of the complex number $z$, respectively. The four complex zeros of $f$ are $\left(u^{0}-\epsilon,-\epsilon\right),\left(u^{0}+\right.$ 
$\epsilon,-\epsilon),\left(u^{0},-\epsilon-i \epsilon\right)$, and $\left(u^{0},-\epsilon+i \epsilon\right)$. Therefore,

$$
\max _{x^{*} \in \mathbb{C}^{2}: f\left(x^{*}\right)=0, y \in[0,1]^{2}}\left\|f^{\prime}\left(x^{*}\right)^{-1} f^{\prime}(y)\right\|=O(1 / \epsilon) .
$$

Moreover,

$$
\omega_{f}=O(1 / \epsilon) .
$$

We now show for the case that $v^{0} \leq \epsilon$ that $B\left(x^{0}, r\right)$ fails the convex hull test if $r \geq O(\epsilon)$. Let $A$ be the circular arc centered at $\left(u^{0},-\epsilon\right)$ that goes from $\left(u^{0}+r, v^{0}-r\right)$ to $\left(u^{0}-r, v^{0}-r\right)$ counterclockwise. Note that $f$ maps $A$ to the circular arc centered at $\left(-\epsilon^{2}, 0\right)$ that goes from $\left(2\left(v^{0}+\epsilon\right) r-2 \epsilon v^{0}-\right.$ $\left.\left(v^{0}\right)^{2}-2 \epsilon^{2}, 2 r\left(v^{0}+\epsilon-r\right)\right)$ to $\left(2\left(v^{0}+\epsilon\right) r-2 \epsilon v^{0}-\left(v^{0}\right)^{2}-2 \epsilon^{2},-2 r\left(v^{0}+\epsilon-\right.\right.$ $r)$ ) counterclockwise. Notice that $2 r\left(v^{0}+\epsilon-r\right) \geq 0$ because $B\left(x^{0}, r\right) \subseteq$ $[0,1]^{2}$. Therefore, the convex hull of $f(A)$ contains the origin if $r>\left(\left(v^{0}\right)^{2}+\right.$ $\left.2 \epsilon v^{0}+2 \epsilon^{2}\right) /\left(2\left(v^{0}+\epsilon\right)\right)=O(\epsilon)$ (recall the assumption that $\left.\epsilon \geq v^{0}\right)$. Since $A \subset B\left(x^{0}, r\right)$, the convex hull of $f\left(B\left(x^{0}, r\right)\right)$ also contains the origin and the convex hull test fails.

\section{Running time analysis}

In this section, we prove a number of theorems relating to the behavior of the KTS algorithm. We first prove the following lemma regarding the product of two polynomials in Bernstein basis.

Lemma 6.1. Let

$$
f(u, v)=\sum_{i=0}^{m} \sum_{j=0}^{n} a_{i j} B_{i, m}(u) B_{j, n}(v), \quad 0 \leq u, v \leq 1
$$

and

$$
g(u, v)=\sum_{i=0}^{m^{\prime}} \sum_{j=0}^{n^{\prime}} a_{i j}^{\prime} B_{i, m^{\prime}}(u) B_{j, n^{\prime}}(v), \quad 0 \leq u, v \leq 1 .
$$

Then

$$
f(u, v) g(u, v)=\sum_{i=0}^{m+m^{\prime}} \sum_{j=0}^{n+n^{\prime}} b_{i j} B_{i, m+m^{\prime}}(u) B_{j, n+n^{\prime}}(v)
$$

where

$$
\left|b_{i j}\right| \leq \max _{i, j}\left|a_{i j}\right| \cdot \max _{i, j}\left|a_{i j}^{\prime}\right|
$$


Proof. Straightforward arithmetic shows that

$$
b_{i j}=\sum_{k=\max \left(0, i-m^{\prime}\right)}^{\min (m, i)} \sum_{l=\max \left(0, j-n^{\prime}\right)}^{\min (n, j)} \frac{\left(\begin{array}{c}
m \\
k
\end{array}\right)\left(\begin{array}{c}
n \\
l
\end{array}\right)\left(\begin{array}{c}
m^{\prime} \\
i-k
\end{array}\right)\left(\begin{array}{c}
n^{\prime} \\
j-l
\end{array}\right)}{\left(\begin{array}{c}
m+m^{\prime} \\
i
\end{array}\right)\left(\begin{array}{c}
n+n^{\prime} \\
j
\end{array}\right)} a_{k l} a_{i-k, j-l}^{\prime} .
$$

Taking absolute value on both sides and bounding $\left|a_{k l}\right|$ (resp. $\left.\left|a_{i-k, j-l}^{\prime}\right|\right)$ with $\max _{i, j}\left|a_{i j}\right|\left(\operatorname{resp} . \max _{i, j}\left|a_{i j}^{\prime}\right|\right)$ gives

$$
\begin{aligned}
\left|b_{i j}\right| & \leq \max \left|a_{i j}\right| \cdot \max \left|a_{i j}^{\prime}\right| \sum_{k=\max \left(0, i-m^{\prime}\right)} \sum_{l=\max \left(0, j-n^{\prime}\right)}^{\min (m, i)} \frac{\left(\begin{array}{c}
m \\
k
\end{array}\right)\left(\begin{array}{c}
n \\
l
\end{array}\right)\left(\begin{array}{c}
m^{\prime} \\
i-k
\end{array}\right)\left(\begin{array}{c}
n^{\prime} \\
j-l
\end{array}\right)}{\left(\begin{array}{c}
m+m^{\prime} \\
i
\end{array}\right)\left(\begin{array}{c}
n+n^{\prime} \\
j
\end{array}\right)} \\
& =\max \left|a_{i j}\right| \cdot \max \left|a_{i j}^{\prime}\right| \sum_{k=\max \left(0, i-m^{\prime}\right)}^{\min (n, j)} \frac{\left(\begin{array}{c}
m \\
k
\end{array}\right)\left(\begin{array}{c}
m^{\prime} \\
i-k
\end{array}\right)}{\left(\begin{array}{c}
m+m^{\prime} \\
i
\end{array}\right)} \sum_{l=\max \left(0, j-n^{\prime}\right)}^{\min (n, j)} \frac{\left(\begin{array}{c}
n \\
l
\end{array}\right)\left(\begin{array}{c}
n^{\prime} \\
j-l
\end{array}\right)}{\left(\begin{array}{c}
n+n^{\prime} \\
j
\end{array}\right)} .
\end{aligned}
$$

However, recall the combinatorial identity

$$
\left(\begin{array}{c}
m+m^{\prime} \\
i
\end{array}\right)=\sum_{k=\max \left(0, i-m^{\prime}\right)}^{\min (m, i)}\left(\begin{array}{c}
m \\
k
\end{array}\right)\left(\begin{array}{c}
m^{\prime} \\
i-k
\end{array}\right) .
$$

Hence, the lemma follows.

We use the above lemma to show a bound on the control points of a Bézier polynomial in term of its function values.

Lemma 6.2. Let $f(u, v):[0,1]^{2} \rightarrow \mathbb{R}^{d}$ be a Bézier polynomial in d dimensions with control points $\left\{\mathbf{b}_{i j}\right\}$

$$
f(u, v)=\sum_{i=0}^{m} \sum_{j=0}^{n} \mathbf{b}_{i j} B_{i, m}(u) B_{j, n}(v), \quad 0 \leq u, v \leq 1 .
$$

The norm of control points of $f$ can be bounded by

$$
\left\|\mathbf{b}_{i j}\right\| \leq c_{m, n} \max _{(u, v): 0 \leq u, v \leq 1}\|f(u, v)\|,
$$

where $c_{m, n}=d m^{m} n^{n}$. 
Remark. An inequality in the other direction, namely, that

$$
\max _{(u, v): 0 \leq u, v \leq 1}\|f(u, v)\| \leq \max \left\|\mathbf{b}_{i j}\right\|,
$$

is a well-known consequence of the convex hull property of Bézier polynomials [4].

Proof. Let $u_{h}=h / m(h=0,1, \ldots, m)$ and $v_{k}=k / n(k=0,1, \ldots, n)$. Define a matrix $A \in \mathbb{R}^{(m+1)(n+1) \times(m+1)(n+1)}$ having element

$$
A_{i j}=B_{r, m}\left(u_{h}\right) B_{s, n}\left(v_{k}\right),
$$

where $h, k, r$, and $s$ are nonnegative integers such that $i-1=h(n+1)+k$, $j-1=r(n+1)+s$, and $0 \leq k, s<n+1$. Define the $(m+1)(n+1)$-by- $d$ matrices

$$
B=\left(\begin{array}{c}
\mathbf{b}_{00}^{T} \\
\mathbf{b}_{01}^{T} \\
\vdots \\
\mathbf{b}_{0 n}^{T} \\
\mathbf{b}_{10}^{T} \\
\vdots \\
\mathbf{b}_{m n}^{T}
\end{array}\right)
$$

and

$$
F=\left(\begin{array}{c}
f\left(u_{0}, v_{0}\right)^{T} \\
f\left(u_{0}, v_{1}\right)^{T} \\
\vdots \\
f\left(u_{0}, v_{n}\right)^{T} \\
f\left(u_{1}, v_{0}\right)^{T} \\
\vdots \\
f\left(u_{m}, v_{n}\right)^{T}
\end{array}\right) .
$$

Observe that

$$
A B=F .
$$

We claim that $A$ is invertible. In particular, we show that the linear system $A \mathbf{x}=\mathbf{e}$ has solution for any arbitrary $\mathbf{e} \in \mathbb{R}^{(m+1)(n+1)}$. Due to the definition of $A$, solving the system $A \mathbf{x}=\mathbf{e}$ is equivalent to finding the control points $\bar{b}_{r s}$ 's of

$$
g(u, v)=\sum_{r=0}^{m} \sum_{s=0}^{n} \bar{b}_{r s} B_{r, m}(u) B_{s, n}(v)
$$


with the property that $g\left(u_{0}, v_{0}\right)=e_{1}, g\left(u_{0}, v_{1}\right)=e_{2}, \ldots, g\left(u_{0}, v_{n}\right)=e_{n+1}$, $g\left(u_{1}, v_{0}\right)=e_{n+2}, \ldots, g\left(u_{m}, v_{n}\right)=e_{(m+1)(n+1)}$. The polynomial $g$ satisfying such property is the Lagrange interpolant

$$
g(u, v)=\sum_{h, k}\left(e_{h(n+1)+k+1} \prod_{r \neq h} \frac{u-u_{r}}{u_{h}-u_{r}} \prod_{s \neq k} \frac{v-v_{s}}{v_{k}-v_{s}}\right) .
$$

Transforming (231) to Bernstein basis yields $\bar{b}_{r s}$ 's, which are the entries of $\mathbf{x}$.

Knowing that $A$ is invertible, we multiply both sides of (21) by $A^{-1}$,

$$
B=A^{-1} F \text {, }
$$

and hence, for any $i=1,2, \ldots, m, j=1,2, \ldots, n$,

$$
\begin{aligned}
\left\|\mathbf{b}_{i j}\right\| & \leq \sum_{k=1}^{d}\left|\left[b_{i j}\right]_{k}\right| \\
& \leq\|B\| \\
& \leq\left\|A^{-1}\right\| \cdot\|F\| \\
& =\left\|A^{-1}\right\| \cdot \max _{0 \leq h \leq m, 0 \leq k \leq n} \sum_{t=1}^{d}\left|f_{t}\left(u_{h}, v_{k}\right)\right| \\
& \leq\left\|A^{-1}\right\| \cdot \max _{0 \leq h \leq m, 0 \leq k \leq n}\left\{d \cdot\left\|f\left(u_{h}, v_{k}\right)\right\|\right\} \\
& \leq d \cdot\left\|A^{-1}\right\| \cdot \max _{(u, v): 0 \leq u, v \leq 1}\|f(u, v)\| .
\end{aligned}
$$

Compare (19) to (25) to see that we can take $c_{m, n}$ to be a scalar that is greater than or equal to $d \cdot\left\|A^{-1}\right\|$.

It remains to compute $\left\|A^{-1}\right\|$. The $j$ th column of $A^{-1}$ is $A^{-1} \mathbf{e}_{j}$, where $\mathbf{e}_{j}$ denotes the $j$ th column of the identity matrix. Let $g_{j}(u, v)$ be a onedimensional Bézier surface with control points $\left\{b_{r s}^{\prime}\right\}$. With similar reasoning as the above,

$$
\left(\begin{array}{c}
b_{00}^{\prime} \\
\vdots \\
b_{m n}^{\prime}
\end{array}\right)=A^{-1}\left(\begin{array}{c}
g_{j}\left(u_{0}, v_{0}\right) \\
\vdots \\
g_{j}\left(u_{m}, v_{n}\right)
\end{array}\right) .
$$

But (26) implies that the $j$ th column of $A^{-1}, A^{-1} \mathbf{e}_{j}$, are the coefficients $b_{r s}^{\prime}$ 's of $g_{j}$ such that, for $h^{\prime}=1,2, \ldots, m$ and $k^{\prime}=1,2, \ldots, n$,

$$
g_{j}\left(u_{h}^{\prime}, v_{k}^{\prime}\right)= \begin{cases}1, & h^{\prime}=h \text { and } k^{\prime}=k, \\ 0, & h^{\prime} \neq h \text { or } k^{\prime} \neq k .\end{cases}
$$


The following Lagrange interpolant $g_{j}$ satisfies (27):

$$
g_{j}(u, v)=\prod_{r \neq h} \frac{u-u_{r}}{u_{h}-u_{r}} \prod_{s \neq k} \frac{v-v_{s}}{v_{k}-v_{s}} .
$$

To bound $\left\|A^{-1}\right\|$, the upper bound of $\left|b_{r s}^{\prime}\right|$ (of $g_{j}$ after transformed to Bernstein basis) is needed. The Bernstein form of $\frac{u-u_{r}}{u_{h}-u_{r}}(r=1, \ldots, h-1, h+$ $1, \ldots, m)$ is

$$
\begin{aligned}
\frac{u-u_{r}}{u_{h}-u_{r}} & =\frac{1-u_{r}}{u_{h}-u_{r}} u-\frac{u_{r}}{u_{h}-u_{r}}(1-u) \\
& =\frac{m-r}{h-r} u-\frac{r}{h-r}(1-u),
\end{aligned}
$$

whose coefficients have absolute value less than or equal to $m$. Similarly, the Bernstein form of $\frac{v-v_{s}}{v_{k}-v_{s}}(s=1, \ldots, k-1, k+1, \ldots, n)$ is

$$
\frac{v-v_{s}}{v_{k}-v_{s}}=\frac{n-s}{k-s} v-\frac{s}{k-s}(1-v)
$$

and its coefficients have absolute value less than or equal to $n$. Applying Lemma 6.1 to (28) shows that

$$
\left|b_{r s}^{\prime}\right| \leq m^{m-1} n^{n-1}
$$

Since (29) holds for any column $j$ of $A^{-1}$ and $A^{-1}$ has $m n$ columns, we have $\left\|A^{-1}\right\| \leq m^{m} n^{n}$.

Using the above lemmas, we analyze the efficiency of KTS by showing that a patch either is a subset of a safe region, passes the Kantorovich test, or passes the exclusion test when it is smaller than a certain size that depends on the condition number of the function. Hence, we have the upper bound of the total number of patches examined by KTS in order to solve the intersection problem.

Recall that the Lipschitz constant $\hat{\omega}$ given by (11) is not the smallest Lipschitz constant of $f^{\prime}\left(x^{0}\right)^{-1} f$ over $D^{\prime}$, where $D^{\prime}$ is given by (8). However, we can show that $\hat{\omega} \leq 4 m^{m} n^{n} \omega$ where $\omega$ denotes the smallest Lipschitz constant of $f^{\prime}\left(x^{0}\right)^{-1} f$ over $D^{\prime}$. Let $\left[\hat{b}_{i j k}\right]_{l}$ 's denote the coefficients of $\hat{g}_{i j k}$. By 


\section{Lemma 6.2.}

$$
\begin{aligned}
\hat{\omega} & \equiv 4 \max _{i, j, k, l}\left|\left[\hat{b}_{i j k}\right]_{l}\right| \\
& \leq 4 m^{m} n^{n} \max _{i, j, k} \max _{x \in X}\left|g_{i j k}^{\prime}(x)\right| \\
& \leq 4 m^{m} n^{n} \max _{x \in X}\left\|g^{\prime}(x)\right\|=4 m^{m} n^{n} \omega .
\end{aligned}
$$

With this bound on $\hat{\omega}$, we can now analyze the behavior of the Kantorovich test.

Theorem 6.3. Let $x^{0}$ be a point in $[0,1]^{2}$ such that $f^{\prime}\left(x^{0}\right)$ is invertible. Let $x^{*}$ be the only zero of $f$ that is contained in $\bar{B}\left(x^{0}, r\right)$, where $r$ is the radius of the patch under consideration. The patch $X=\bar{B}\left(x^{0}, r\right) \subseteq[0,1]^{2}$ passes the Kantorovich test if

$$
r \leq \frac{9-4 \sqrt{5}}{m^{m} n^{n} \omega_{D^{\prime}}}
$$

Proof. The first step is to show that $\eta \hat{\omega} \leq 1 / 4$, where $\hat{\omega}$ is as in (11). Since $r \leq 1 / 2, \bar{B}\left(x^{0}, 2 \epsilon(m, n) r\right) \subseteq D^{\prime}$. Observe that for any $x, y \in D^{\prime}$,

$$
\begin{aligned}
\left\|f^{\prime}\left(x^{0}\right)^{-1}\left(f^{\prime}(x)-f^{\prime}(y)\right)\right\|= & \|\left(f^{\prime}\left(x^{*}\right)^{-1}+\left(f^{\prime}\left(x^{0}\right)^{-1}-f^{\prime}\left(x^{*}\right)^{-1}\right)\right) \\
& \left(f^{\prime}(x)-f^{\prime}(y)\right) \| \\
\leq & \left\|f^{\prime}\left(x^{*}\right)^{-1}\left(f^{\prime}(x)-f^{\prime}(y)\right)\right\|+\| f^{\prime}\left(x^{*}\right)^{-1} \\
& \left(f^{\prime}\left(x^{*}\right)-f^{\prime}\left(x^{0}\right)\right) f^{\prime}\left(x^{0}\right)^{-1}\left(f^{\prime}(x)-f^{\prime}(y)\right) \| \\
\leq & \omega_{D^{\prime}}\|x-y\|+\left\|f^{\prime}\left(x^{*}\right)^{-1}\left(f^{\prime}\left(x^{*}\right)-f^{\prime}\left(x^{0}\right)\right)\right\| . \\
& \left\|f^{\prime}\left(x^{0}\right)^{-1}\left(f^{\prime}(x)-f^{\prime}(y)\right)\right\| \\
\leq & \omega_{D^{\prime}}\|x-y\|+ \\
& \omega_{D^{\prime}}\left\|x^{*}-x^{0}\right\| \cdot\left\|f^{\prime}\left(x^{0}\right)^{-1}\left(f^{\prime}(x)-f^{\prime}(y)\right)\right\| \\
\leq & \omega_{D^{\prime}}\|x-y\|+ \\
& \omega_{D^{\prime}} r \cdot\left\|f^{\prime}\left(x^{0}\right)^{-1}\left(f^{\prime}(x)-f^{\prime}(y)\right)\right\| .
\end{aligned}
$$

Since (31) implies $\omega_{D^{\prime}} r \leq(9-4 \sqrt{5}) /\left(m^{m} n^{n}\right) \leq 9-4 \sqrt{5}$, we have that

$$
1-\omega_{D^{\prime}} r \geq 4(\sqrt{5}-2) \text {. }
$$

Since $1-\omega_{D^{\prime}} r>0$, (32) becomes

$$
\left\|f^{\prime}\left(x^{0}\right)^{-1}\left(f^{\prime}(x)-f^{\prime}(y)\right)\right\| \leq\left(\frac{\omega_{D^{\prime}}}{1-\omega_{D^{\prime}} r}\right)\|x-y\| .
$$


Hence

$$
\omega \leq \frac{\omega_{D^{\prime}}}{1-\omega_{D^{\prime}} r}
$$

where $\omega$ is the smallest Lipschitz constant of $f^{\prime}\left(x^{0}\right)^{-1} f$ over $D^{\prime}$. Therefore, by (30),

$$
\begin{aligned}
\hat{\omega} & \leq \frac{4 m^{m} n^{n} \omega_{D^{\prime}}}{1-\omega_{D^{\prime}} r} \\
& \leq \frac{4 m^{m} n^{n} \omega_{D^{\prime}}}{1-\frac{9-4 \sqrt{5}}{m^{m} n^{n}}} \\
& \leq \frac{4 m^{m} n^{n} \omega_{D^{\prime}}}{1-(9-4 \sqrt{5})} \\
& =\frac{m^{m} n^{n} \omega_{D^{\prime}}}{\sqrt{5}-2} .
\end{aligned}
$$

Recall that $f\left(x^{*}\right)=0$ and $X \subseteq D^{\prime}$. Observe that

$$
\begin{aligned}
\eta & \equiv\left\|f^{\prime}\left(x^{0}\right)^{-1} f\left(x^{0}\right)\right\| \\
& =\left\|f^{\prime}\left(x^{0}\right)^{-1}\left(f\left(x^{0}\right)-f\left(x^{*}\right)\right)\right\| \\
& \leq\left(\max _{x \in X}\left\|f^{\prime}\left(x^{0}\right)^{-1} f^{\prime}(x)\right\|\right) \cdot\left\|x^{0}-x^{*}\right\| \\
& \leq\left(\max _{x \in X}\left\|f^{\prime}\left(x^{0}\right)^{-1}\left(f^{\prime}(x)-f^{\prime}\left(x^{0}\right)\right)+f^{\prime}\left(x^{0}\right)^{-1} f^{\prime}\left(x^{0}\right)\right\|\right) \cdot r \\
& \leq\left(\max _{x \in X}\left\|f^{\prime}\left(x^{0}\right)^{-1}\left(f^{\prime}(x)-f^{\prime}\left(x^{0}\right)\right)\right\|+1\right) \cdot r \\
& \leq(\omega r+1) r \\
& \leq \frac{r}{1-\omega_{D^{\prime}} r} .
\end{aligned}
$$

By (31) and (33), the above becomes

$$
\eta \leq \frac{9-4 \sqrt{5}}{4(\sqrt{5}-2) m^{m} n^{n} \omega_{D^{\prime}}} .
$$

The bounds (34) and (35) together give

$$
\eta \hat{\omega} \leq \frac{1}{4}
$$


The last step is to to verify the other condition that $\bar{B}\left(x^{0}, \rho_{-}\right) \subseteq \bar{B}\left(x^{0}, 2 \epsilon(m, n) r\right)$. Noting that $\sqrt{1-2 h} \geq 1-2 h$ for $0 \leq h \leq 1 / 2$, it is seen that

$$
\begin{aligned}
\rho_{-}(\eta, \hat{\omega}) & =\frac{1-\sqrt{1-2 \eta \hat{\omega}}}{\hat{\omega}} \\
& \leq 2 \eta \\
& \leq \frac{2 r}{1-\omega_{D^{\prime}}} \\
& \leq \frac{2 r}{1-\frac{9-4 \sqrt{5}}{m^{m} n^{n}}} \\
& =2 \epsilon(m, n) r . \quad \square
\end{aligned}
$$

Next results are concerned with the size of the patch satisfying the exclusion test.

Lemma 6.4. Let $f: \mathbb{C}^{n} \rightarrow \mathbb{C}^{n}$ be a polynomial function with generic coefficients. Assume that all zeros of $f$ are invertible. Let $x^{0}$ be a point in $\mathbb{R}^{n}$. If

$$
\left\|f^{\prime}\left(x^{*}\right)^{-1} f\left(x^{0}\right)\right\| \leq \frac{1}{2 \omega_{f}}
$$

for all complex zeros $x^{*}$ of $f$, then there exists $\hat{x}^{*}$, a zero of $f$, such that

$$
\begin{aligned}
\left\|x^{0}-\hat{x}^{*}\right\| & \leq \frac{1-\sqrt{1-2 \omega_{f}\left\|f^{\prime}\left(\hat{x}^{*}\right)^{-1} f\left(x^{0}\right)\right\|}}{\omega_{f}} \\
& \equiv \sigma\left(\hat{x}^{*}, x^{0}\right) .
\end{aligned}
$$

Proof. By the assumption that $f$ has generic coefficients, the polynomial $f$ has a finite number of zeros. Let $x_{1}^{*}, x_{2}^{*}, \ldots, x_{d}^{*}$ be all the complex zeros of $f$. Recall that a multiple zero has singular Jacobian. Hence, $f$ has no multiple zeros by assumption.

Define the polynomial $\bar{f}(x)=f(x)-f\left(x^{0}\right)$. Note that $x^{0}$ is a zero of $\bar{f}$. We apply the Kantorovich's theorem for complex functions (see [5]) to each $x_{i}^{*}$ with respect to $\bar{f}$. For each $x_{i}^{*}$, we use $D=\bar{B}\left(x_{i}^{*}, \rho_{*}\left(x_{i}^{*}\right)\right)$ and $\omega=\omega_{f}$. Since $\eta \equiv\left\|\bar{f}^{\prime}\left(x_{i}^{*}\right)^{-1} \bar{f}\left(x_{i}^{*}\right)\right\|=\left\|f^{\prime}\left(x_{i}^{*}\right)^{-1} f\left(x^{0}\right)\right\|$, the assumption (36) guarantees that the condition $\eta \omega \leq 1 / 2$ is satisfied. The condition $\bar{B}\left(x_{i}^{*}, \rho_{-}\right) \subseteq D$ is also satisfied by the definition of $D$. Therefore, the Kantorovich theorem states that there is a zero of $\bar{f}$, call it $\bar{x}_{i}^{*}$, such that

$$
\left\|\bar{x}_{i}^{*}-x_{i}^{*}\right\| \leq \sigma\left(x_{i}^{*}, x^{0}\right)
$$


Recall that, for any $j, x_{j}^{*}$ is the unique zero of $f$ in $\bar{B}\left(x_{j}^{*}, \rho_{*}\left(x_{j}^{*}\right)\right)$. Therefore,

$$
\left\|x_{i}^{*}-x_{j}^{*}\right\|>\max \left\{\rho_{*}\left(x_{i}^{*}\right), \rho_{*}\left(x_{j}^{*}\right)\right\}, i \neq j .
$$

But (38) and (39) together imply that

$$
\bar{x}_{i}^{*} \neq \bar{x}_{j}^{*}, i \neq j .
$$

Hence the mapping $x_{i}^{*} \rightarrow \bar{x}_{i}^{*}$ is injective. But since $f$ has generic coefficients and $f$ and $\bar{f}$ are of the same degrees, $f$ has at least as many zeros as $\bar{f}[3$. This implies that $x^{0}=\bar{x}_{i}^{*}$, for some $i$. The lemma follows.

Theorem 6.5. Let $f(x)=f(u, v)$ be a Bézier polynomial in two dimensions with generic control points $\left\{\mathbf{b}_{i j}\right\}$. Let $m$ be the highest $u$ degree of $f$ and $n$ be its highest $v$ degree. Let $x^{0}=\left(u^{0}, v^{0}\right)$ be a point in $[0,1]^{2}$ such that $f^{\prime}\left(x^{0}\right)$ is invertible and $f\left(x^{0}\right) \neq 0, x^{*}$ be the closest zero in $\mathbb{R}^{2}$ of $f$ to $x^{0}$, and $\delta$ denote $\left\|x^{0}-x^{*}\right\|$. Let $r>0$ be such that $\bar{B}\left(x^{0}, r\right) \subseteq[0,1]^{2}$. Assume $\delta>\frac{1}{\omega_{f}}$. Define $\hat{f}(\hat{u}, \hat{v})$ such that

$$
\hat{f}(\hat{u}, \hat{v})=f\left(2 r \hat{u}+u^{0}-r, 2 r \hat{v}+v^{0}-r\right) .
$$

In other word, $\hat{f}$ is a Bézier polynomial that reparametrizes with $[0,1]^{2}$ the Bézier patch defined by $f$ over the patch $\bar{B}\left(x^{0}, r\right)$. The convex hull of the control points of $\hat{f}$ does not contain the origin if

$$
r \leq \frac{1}{2 c_{m, n} \operatorname{cond}(f)^{2}}
$$

where $c_{m, n}=2 m^{m} n^{n}$.

Proof. Let $X$ denote the patch $\bar{B}\left(x^{0}, r\right)$ and $x$ denote an arbitrary point in $X$. Since $\delta>\frac{1}{\omega_{f}}$, the contrapositive of Lemma 6.4 implies there exists a zero $\bar{x}^{*}$ of $f$ satisfying $\left\|f^{\prime}\left(\bar{x}^{*}\right)^{-1} f\left(x^{0}\right)\right\|>\frac{1}{2 \omega_{f}}$. Therefore, the condition (42) implies

$$
\begin{aligned}
r & \leq \frac{1}{2 c_{m, n} \operatorname{cond}(f)^{2}} \\
& \leq \frac{1}{2 c_{m, n} \omega_{f}\left\|f^{\prime}\left(\bar{x}^{*}\right)^{-1} f^{\prime}(x)\right\|} \\
& <\frac{\left\|f^{\prime}\left(\bar{x}^{*}\right)^{-1} f\left(x^{0}\right)\right\|}{c_{m, n}\left\|f^{\prime}\left(\bar{x}^{*}\right)^{-1} f^{\prime}(x)\right\|} .
\end{aligned}
$$


More specifically, we have

$$
r<\frac{\left\|f^{\prime}\left(\bar{x}^{*}\right)^{-1} f\left(x^{0}\right)\right\|}{c_{m, n} \max _{y \in X}\left\|f^{\prime}\left(\bar{x}^{*}\right)^{-1} f^{\prime}(y)\right\|},
$$

which is equivalent to

$$
c_{m, n} \cdot \max _{y \in X}\left\|f^{\prime}\left(\bar{x}^{*}\right)^{-1} f^{\prime}(y)\right\| \cdot r<\left\|f^{\prime}\left(\bar{x}^{*}\right)^{-1} f\left(x^{0}\right)\right\| .
$$

Recall that $\max _{y \in X}\left\|f^{\prime}\left(\bar{x}^{*}\right)^{-1} f^{\prime}(y)\right\|$ is the Lipschitz constant for $f^{\prime}\left(\bar{x}^{*}\right)^{-1} f$ on $X$. Hence, for any $x \in X$,

$$
\begin{aligned}
\left\|f^{\prime}\left(\bar{x}^{*}\right)^{-1} f(x)-f^{\prime}\left(\bar{x}^{*}\right)^{-1} f\left(x^{0}\right)\right\| & \leq \max _{y \in X}\left\|f^{\prime}\left(\bar{x}^{*}\right)^{-1} f^{\prime}(y)\right\| \cdot\left\|x-x^{0}\right\| \\
& \leq \max _{y \in X}\left\|f^{\prime}\left(\bar{x}^{*}\right)^{-1} f^{\prime}(y)\right\| \cdot r .
\end{aligned}
$$

Combining (43) and (44) gives

$$
c_{m, n} \cdot\left\|f^{\prime}\left(\bar{x}^{*}\right)^{-1} f(x)-f^{\prime}\left(\bar{x}^{*}\right)^{-1} f\left(x^{0}\right)\right\|<\left\|f^{\prime}\left(\bar{x}^{*}\right)^{-1} f\left(x^{0}\right)\right\|,
$$

which is equivalent to

$$
c_{m, n} \cdot\left\|f^{\prime}\left(\bar{x}^{*}\right)^{-1} \hat{f}(\hat{x})-f^{\prime}\left(\bar{x}^{*}\right)^{-1} \hat{f}\left(\hat{x}^{0}\right)\right\|<\left\|f^{\prime}\left(\bar{x}^{*}\right)^{-1} \hat{f}\left(\hat{x}^{0}\right)\right\|
$$

for some $\hat{x} \in[0,1]^{2}$, where $\hat{x}$ is the rescaled $x$ and $\hat{x}^{0}$ is the rescaled $x^{0}$ according to (41). In particular,

$$
c_{m, n} \cdot \max _{\hat{x} \in[0,1]^{2}}\left\|f^{\prime}\left(\bar{x}^{*}\right)^{-1} \hat{f}(\hat{x})-f^{\prime}\left(\bar{x}^{*}\right)^{-1} \hat{f}\left(\hat{x}^{0}\right)\right\|<\left\|f^{\prime}\left(\bar{x}^{*}\right)^{-1} \hat{f}\left(\hat{x}^{0}\right)\right\| .
$$

Applying Lemma 6.2 to the Bézier surface patch $f^{\prime}\left(\bar{x}^{*}\right)^{-1} \hat{f}-f^{\prime}\left(\bar{x}^{*}\right)^{-1} \hat{f}\left(\hat{x}^{0}\right)$ yields, for any control points $\overline{\mathbf{b}}_{i j}$ of $h \equiv f^{\prime}\left(\bar{x}^{*}\right)^{-1} \hat{f}$,

$$
\left.\left\|\overline{\mathbf{b}}_{i j}-h\left(\hat{x}^{0}\right)\right\| \leq c_{m, n} \cdot \max _{\hat{x} \in[0,1]^{2}} \| h(\hat{x})-h\left(\hat{x}^{0}\right)\right) \| .
$$

Substituting (46) into the left hand side of (45) yields

$$
\left\|\overline{\mathbf{b}}_{i j}-h\left(\hat{x}^{0}\right)\right\|<\left\|h\left(\hat{x}^{0}\right)\right\|
$$

which implies that the convex hull of $\left\{\overline{\mathbf{b}}_{i j}\right\}$ does not contain the origin. Since $f^{\prime}\left(\bar{x}^{*}\right)^{-1}$ is invertible, the convex hull of the control points of $\hat{f}$ does not contain the origin, either. 
Theorem 6.6. Let $f(x)=f(u, v)$ be a Bézier polynomial in two dimensions with generic control points $\left\{\mathbf{b}_{i j}\right\}$ whose zeros are sought. Let $X=\bar{B}\left(x^{0}, r\right)$ be a patch under consideration during the course of the KTS algorithm. The algorithm does not need to subdivide $X$ if

$$
r \leq \frac{1}{2} \cdot \min \left\{\frac{9-4 \sqrt{5}}{m^{m} n^{n} \omega_{D^{\prime}}}, \frac{1}{2 c_{m, n} \operatorname{cond}(f)^{2}}\right\} .
$$

Proof. If $X$ contains a zero $x^{*}$, (48) implies that $\bar{B}\left(x^{0}, r\right)$ passes the Kantorovich test according to Theorem 6.3. If $X$ does not contain a zero and $\delta>\frac{1}{\omega_{f}}$, where $\delta$ is the distance between $x^{0}$ and the closest zero $x^{*}$, (48) implies that $X$ is excluded by the exclusion test according to Theorem 6.5.

Observe that $\omega_{*} \leq \omega_{f}$. If $X$ does not contain a zero but $\delta \leq \frac{1}{\omega_{f}}$, for any $x \in X$,

$$
\begin{aligned}
\left\|x-x^{*}\right\| & \leq\left\|x-x^{0}\right\|+\left\|x^{0}-x^{*}\right\| \\
& \leq r+\delta \\
& \leq \frac{9-4 \sqrt{5}}{\omega_{f}}+\frac{1}{\omega_{f}} \\
& <\frac{2}{\omega_{f}} \leq \frac{2}{\omega_{*}}=\rho_{*} .
\end{aligned}
$$

In other word, $X$ is contained within $\bar{B}\left(x^{*}, \rho_{*}\right)$, a safe region and therefore is excluded, provided that $x^{*}$ is found before $X$ is checked against all safe regions. By Theorem [6.3, $x^{*}$ is found by a region of size $2 r \leq$ $(9-4 \sqrt{5}) /\left(m^{m} n^{n} \omega_{D^{\prime}}\right)$. Since KTS examines larger regions before smaller ones, $x^{*}$ is found before $X$ is checked against safe regions.

\section{Computational results}

The KTS algorithm is implemented in Matlab and is tested against a number of problem instances with varying condition numbers. The degrees of the test surfaces are between biquadratic and quartic. For the experiment, we use the algorithm by Jónsson and Vavasis [9] to compute the complex zeros required to estimate the condition number. Table 1 compares the efficiency of KTS for each case with its condition number. The total number of subpatches examined by KTS during the entire computation and the width of the smallest patch among those examined are reported. 


\begin{tabular}{|r|r|r|}
\hline $\operatorname{cond}(f)$ & Number of patches examined & Smallest width \\
\hline \hline $6.2 \times 10^{2}$ & 13 & .125 \\
$2.2 \times 10^{3}$ & 17 & .0625 \\
$7.0 \times 10^{4}$ & 17 & .0625 \\
$2.9 \times 10^{5}$ & 29 & .0625 \\
$1.5 \times 10^{6}$ & 25 & .015625 \\
$2.2 \times 10^{7}$ & 57 & .0313 \\
$3.5 \times 10^{9}$ & 149 & .0039 \\
$2.9 \times 10^{11}$ & 257 & .0039 \\
\hline
\end{tabular}

Table 1: Efficiency of KTS algorithm on line-surface intersection problems of different condition numbers.

\section{Conclusion and future directions}

We present KTS algorithm for finding the intersections between a Bézier surface and a line. By using the combination of subdivision with bounding convex hull and Kantorovich's theorem, our algorithm can take advantage of the quadratic convergence of Newton's method without the problems of divergence and missing some intersections that commonly occur with Newton's method. We also show that the efficiency of KTS depends solely on the conditioning of the problem. Nevertheless, there are a number of questions left unanswered by this article such as

- Extensibility to piecewise polynomial surfaces and/or NURBS. Since KTS only requires the ability to compute the convex hull bounding volume of a subpatch and to estimate the Lipschitz constant up to a constant factor of the smallest Lipschitz constant, it may be possible to extend KTS to handle these more general surfaces if these estimates can be computed relatively quickly.

- Tighter condition number. The condition number presented earlier seems overly loose. It is likely that a tighter condition number exists. If a tighter condition number is found, we would be able to calculate a tighter bound on the running time of KTS, too.

- The necessity of the generic coefficients assumption. Is it possible to analyze the efficiency of KTS without this assumption? 
- Using KTS in floating point arithmetic. In the presence of roundoff error, we may need to make adjustments for KTS to be able to guarantee that all zeros are found. In addition, the accuracy of the computed zeros would become an important issue in this case.

\section{References}

[1] W. Barth and W. Stürzlinger. Efficient ray-tracing for Bézier and Bspline surfaces. Computers and Graphics, 17(4):423-430, 1993.

[2] Peter Deuflhard and Gerhard Heindl. Affine invariant convergence theorems for Newton's method and extensions to related methods. SIAM J. Numer. Anal., 16:1-10, 1980.

[3] I. Z. Emiris and J. F. Canny. Efficient incremental algorithms for the sparse resultant and the mixed volume. J. Symb. Comput., 20:117-149, 1995.

[4] Gerald Farin. Curves and Surfaces for CAGD: A Practical Guide, fifth edition, Academic Press, 2002.

[5] Rida T. Farouki, Bethany K. Kuspa, Carla Manni, and Alessandra Sestini. Efficient solution of the complex quadratic tridiagonal system for $\mathcal{C}^{2}$ PH quintic splines. Numerical Algorithms, 27:35-60, 2001.

[6] Alain Fournier and John Buchanan. Chebyshev polynomials for boxing and intersections of parametric curves and surfaces. Computer Graphics Forum, 13(3):C-127-C-142, 1994.

[7] Robert M. Freund and Jorge R. Vera. Condition-Based Complexity of Convex Optimization in Conic Linear Form via the Ellipsoid Algorithm. SIAM J. Optim., 10:155-176, 1999.

[8] Gene H. Golub and Charles F. Van Loan. Matrix Computations, third edition, the Johns Hopkins University Press, 1996.

[9] G. Jónsson and S. Vavasis. Accurate solution of polynomial equations using Macaulay resultant matrices. Mathematics of Computation, 74:221262, 2005. 
[10] James T. Kajiya. Ray tracing parametric patches. Computer Graphics (SIGGRAPH '82 Proceedings), 16(3):245-254, 1982.

[11] L. Kantorovich. On Newton's method for functional equations (Russian). Dokl. Akad. Nauk SSSR, 59:1237-1240, 1948.

[12] Daniel Lischinski and Jakob Gonczarowski. Improved techniques for ray tracing parametric surfaces. The Visual Computer, 6:134-152, 1990.

[13] T. Nishita, T. W. Sederberg, and M. Kakimoto. Ray tracing trimmed rational surface patches. ACM Computer Graphics, 24(4):337-345, 1990.

[14] K. H. Qin, M. L. Gong, Y. J. Guan, and W. P. Wang. A new method for speeding up ray tracing NURBS surfaces. Computers and Graphics, 21(5):577-586, 1997.

[15] Seven M. Rubin and Turner Whitted. A 3-dimensional representation for fast rendering of complex scenes. Computer Graphics (SIGGRAPH '80 Proceedings), 14(3):110-116, 1980.

[16] E. C. Sherbrooke and N. M. Patrikalakis. Computation of the solutions of nonlinear polynomial systems. Computer Aided Geometric Design, 10(5):379-405, 1993.

[17] M. A. J. Sweeney and R. H. Bartels. Ray tracing free-form B-spline surface. IEEE Computer Graphics and Application, 6:41-49, 1986.

[18] Kim-Chuan Toh and Lloyd N. Trefethen. Calculation of pseudospectra by the Arnoldi iteration. SIAM J. Sci. Comput., 17:1-15, 1996.

[19] Daniel L. Toth. On ray tracing parametric surfaces. Computer Graphics (SIGGRAPH '85 Proceedings), 19(3):171-179, 1985.

[20] S. W. Wang, Z. C. Shih, and R. C. Chang. An efficient and stable ray tracing algorithm for parametric surfaces. Journals of Information Science and Engineering, 18(4):541-561, 2002.

[21] Turner Whitted. An improved illumination model for shaded display. Communications of the ACM, 23(6):343-349, 1980.

[22] C. G. Yang. On speeding up ray tracing of B-spline surfaces. Computer Aided Design, 19:122-130, 1987. 